\title{
Criatividade e Multiculturalismo: Revisão de Literatura
}

\author{
Marina Porto Ribeiro, * \\ Orcid.org/0000-0002-8634-9240 \\ Denise de Souza Fleith ${ }^{1}$ \\ Orcid.org/0000-0001-7512-8023
}

${ }^{1}$ Universidade de Brasília, Brasília, DF, Brasil

\section{Resumo}

Este estudo examinou publicações em periódicos, no período de 2010 a 2015, sobre a relação entre multiculturalismo e criatividade. O levantamento foi realizado nas bases de dados Capes, PsycNET e SciELO. Foram selecionados 30 artigos que atenderam aos critérios de busca pré-estabelecidos. Os artigos foram analisados quanto às categorias: periódico, países de produção, objetivo de pesquisa, tipo de autoria, ano de publicação, tipo de estudo, abordagem do estudo, população pesquisada e instrumentos utilizados. Observou-se aumento das publicações sobre a temática nos últimos anos. Pesquisadores de regiões de alto fluxo migratório, como Estados Unidos, Europa e Ásia, demonstraram maior interesse pela área. Não houve publicação brasileira, tampouco latina. A maioria dos estudos encontrados era empírico, de abordagem quantitativa e investigou participantes adultos. Os estudos revisados indicaram, de forma geral, haver relação entre criatividade e multiculturalismo, mediada por mecanismos psicológicos e condições do ambiente.

Palavras-chave: Criatividade, multiculturalismo, biculturalismo.

\section{Creativity and Multiculturalism: Literature Review}

\begin{abstract}
This study examined journals articles, published from 2010 to 2015, about the relationship between multiculturalism and creativity. A literature search was conducted in the databases Capes, PsycNET and SciELO. Thirty articles that fit within pre-established inclusion criteria were selected. The articles were analyzed according to the categories: journal, country of production, target, sample, and instruments. There was an increase in the number of publications concerning this theme in the last years. Researchers from regions with high migratory flow, such as United States, Europe and Asia, seemed more interested in the matter. There was no publication neither from Brazil nor Latin America. The majority of the studies selected were empirical, quantitative, and used adult samples. The reviewed studies indicated a relationship between creativity and multiculturalism, mediated by psychological mechanisms and environmental conditions.
\end{abstract}

Keywords: Creativity, multiculturalism, biculturalism.

Endereço para correspondência: Universidade de Brasília, Instituto de Psicologia, Departamento de Psicologia Escolar e do Desenvolvimento, Campus Universitário, Asa Norte, Brasília, DF, Brasil 70910-900. Fone: (61) 3107-6830. Fax: (61) 3273-6378. E-mail: marinaportopsi@gmail.com 


\section{Creatividad y Multiculturalismo: Revisión de la Literatura}

\section{Resumen}

Este estudio examinó publicaciones científicas en revistas en el periodo de 2010 a 2015, sobre la relación entre multiculturalismo y creatividad. La búsqueda fue realizada en las bases de datos Capes, PsycNET y SciELO. Fueron seleccionados 30 artículos que cumplieron con los criterios de búsqueda preestablecidos. Los artículos fueron analizados de acuerdo con las categorías: revista, países de producción, objetivo de la investigación, tipo de autoría, año de publicación, tipo de estudio, enfoque de estudio, población investigada e instrumentos utilizados. Se observó un aumento de las publicaciones sobre el tema en los últimos años. Los investigadores de las regiones de alta migración, como Estados Unidos, Europa y Asia han demostrado mayor interés en el tema. No se encontraron publicaciones brasileñas o latinas. La mayoría de los estudios fueron empíricos, cuantitativos y con participantes adultos. Los estudios revisados indican, en general, que existe una relación entre creatividad y multiculturalismo, mediada por los mecanismos psicológicos y las condiciones ambientales.

Palabras clave: Creatividad, multiculturalismo, biculturalismo.

O multiculturalismo é um fenômeno crescente e multifacetado. Inglis (1996) chama a atenção para a popularização do termo multiculturalismo e aponta três referências distintas, mas inter-relacionados para seu uso: demográfica-descritiva, programática-política e ideológica-normativa. O emprego do termo multiculturalismo na perspectiva demográfica-descritiva reflete a existência de diversas raças ou etnias em uma sociedade, uma miscigenação cultural que produz significados sociais. Esse será o foco do presente estudo. Já a aplicação programática-política do conceito remete a programas e políticas estabelecidas para lidar com a diversidade étnica ou racial. O uso ideológico-normativo constitui um modelo baseado na teoria sociológica e na consideração ético-filosófica que enfatiza a existência da diversidade étnica e a necessidade de assegurar aos indivíduos oriundos de culturas não dominantes acesso equitativo aos princípios constitucionais e aos valores compartilhados na sociedade.

Indivíduos que vivenciam experiências multiculturais demográficas, trabalhando, estudando ou convivendo em ambientes variados, começam, à medida em que se engajam à nova cultura, a avaliar e questionar suas próprias crenças, costumes e tradições (Tadmor, Hong, Chao, Wiruchnipawan, \& Wang, 2012). É cada vez é maior o número de pessoas que se identificam com culturas diferentes da sua (Mok \&
Morris, 2012). Sam e Berry (2010) destacam que refugiados, requerentes de asilo, imigrantes, expatriados, indígenas e minorias étnicas são as amostras mais estudadas na pesquisa sobre identidades multiculturais. Desde os anos 50, Malzberg e Lee (1956) já pesquisavam adversidades sociais e psicológicas decorrentes dos processos de identificação multicultural.

Estudos mais recentes têm revelado implicações bastante positivas desse tipo de experiência. Diversos benefícios já foram relacionados ao multiculturalismo, entre eles: adaptabilidade, liderança e capacidade de resolução de problemas (Friedman \& Liu, 2009); sucesso profissional (Tadmor, Galinsky, \& Maddux, 2012); ampliação de redes sociais (Mok, Morris, Benet-Martínez, \& Karakitapoglu-Aygun, 2007); e criatividade (Crisp \& Turner, 2011; Leung \& Chiu, 2010; Saad, Damian, Benet-Martínez, Moons, \& Robins, 2012). A criatividade, entendida como um processo por meio do qual algo útil e original é criado (Amabile, 1996), tem sido amplamente investigada nessa relação. Em um artigo teórico, Goclowska e Crisp (2014) listaram mais de 10 estudos publicados entre os anos de 2008 e 2012 sobre criatividade e multiculturalismo.

Diante do contraste multicultural, o que levaria o indivíduo a desenvolver ou não características e competências associadas à criatividade? A adaptação psicológica de migrantes é um fenômeno bastante explorado, especialmente 
pela comunidade científica internacional (Kuo, 2014, Sam \& Berry, 2010; Yoon et al., 2012). Pesquisadores estudaram diversos aspectos, desde características dos indivíduos e dos grupos étnicos até atributos e atitudes dos residentes no país hospedeiro (Aronowitz, 1984; Berry \& Sam, 1997).

Berry (1990), Berry, Kim e Boski (1987), Berry, Kim, Minde e Mok (1987), Berry e Sam (1997) investigaram a adaptação multicultural com base nos comportamentos e atitudes que o indivíduo adota no que concerne à sua cultura natal e à nova cultura. Segundo esses autores, as pessoas podem identificar-se com ambas, nenhuma ou apenas uma das culturas, por meio de um processo que foi denominado aculturação. São quatro as estratégias apontadas: assimilação, separação, marginalização e integração.

Tadmor, Galinsky et al. (2012) verificaram que estudantes universitários que adotaram estratégia aculturativa de integração tinham escores superiores em medidas de criatividade em comparação a indivíduos que adotaram estratégia aculturativa de separação ou assimilação. Dessa forma, o modelo de identificação multicultural baseado nas estratégias de aculturação explicaria como o multiculturalismo relaciona-se à criatividade.

No entanto, Benet-Martínez, Leu, Lee e Morris (2002) apontam lacunas na concepção do conceito de identidade bicultural. Para os autores, a teoria das estratégias de aculturação desconsidera a forma como as pessoas lidam com as duas culturas na estratégia de integração e a percepção paradoxal de pertencer a duas culturas e, ao mesmo tempo, a nenhuma (Benet-Martínez \& Haritatos, 2005). A partir das críticas, Benet-Martínez et al. (2002) delinearam um novo construto teórico para investigar diferenças individuais nas formas de organização das identidades biculturais, desta feita focando apenas na estratégia de aculturação integrativa.

A Integração da Identidade Bicultural ou Bicultural Identity Integration (BII) foi proposta para medir o quanto os indivíduos biculturais percebem suas identidades compatíveis e integradas ou opostas e difíceis de integrar (Benet-Martínez et al., 2002). Há alta BII quando o in- divíduo percebe suas duas identidades culturais como interconectadas, coerentes ou integradas, e baixa BII quando as percebe como opostas, separadas ou desintegradas (Benet-Martínez \& Haritatos, 2005). De forma análoga ao que ocorreu na pesquisa que considerou as estratégias de aculturação, a criatividade também estaria relacionada ao processo de identificação multicultural descrito pelo modelo BII. Pessoas biculturais com alta BII tendem a ser mais criativas do que as com baixa BII (Cheng, Sanchez-Burks, \& Lee, 2008; Saad et al., 2012).

Além dessas duas possíveis explicações sobre a relação entre multiculturalismo e criatividade, baseadas na identificação do indivíduo com as culturas, outras teorias foram propostas. Goclowska e Crisp (2014) sumarizaram investigações dessa vertente e propuseram um modelo teórico para explicar o ganho criativo de pessoas que se identificam com dois estratos culturais (biculturais). Os autores descreveram três componentes essenciais à identificação cultural: (a) aprofundamento, que implicaria grau de envolvimento cultural, aprendizagem e processamento de informações relacionadas ao novo papel desempenhado na cultura anfitriã; (b) duplo engajamento, que envolveria desenvolver a capacidade de ver o mundo por meio de diferentes identidades sociais, o que traria benefícios para o pensamento flexível e criativo; e (c) distância cultural, que representaria a dimensão em que valores, costumes e características predominantes nos dois grupos divergem e, consequentemente, exigem que o indivíduo elabore soluções complexas integrativas. O efetivo pertencimento a dois grupos culturalmente distantes, vivenciado de forma aprofundada, exigiria do indivíduo desenvolvimento de maneiras inovadoras de pensar e agir, o que fomentaria o potencial criativo (Goclowska \& Crisp, 2014).

Há ainda hipóteses de que o estado emocional, positivo ou negativo (Cheng, Leung, \& Wu, 2011), e o clima para a inclusão (Li, Lin, Tien, \& Chen, 2015) sejam moderadores da relação entre criatividade e multiculturalismo. Não foi identificado na literatura, até o presente momento, um consenso de como o multiculturalismo pode possibilitar o desenvolvimento da criatividade, mas 
ficam claros os esforços envidados para investigar essa relação.

Com base nessa argumentação, é relevante avaliar o que já foi explorado e descoberto, especialmente nos últimos anos, buscando-se compreender os avanços das pesquisas nessa seara e identificar as necessidades de pesquisas futuras. Segundo Witter (1999), a análise de produções científicas contribui para o aprimoramento do conhecimento disponível, sinalizando o que pesquisadores têm demonstrado mais interesse, tendências teóricas e metodológicas, amostras mais estudadas e outros aspectos.

No Brasil, a relação entre criatividade e multiculturalismo ainda é pouco investigada. Existem muitos estudos brasileiros sobre criatividade, inclusive revisões de literatura (Nakano, 2005; Nakano \& Wechsler, 2007; Wechsler \& Nakano, 2003; Zanella \& Titon, 2005), mas a associação desse construto ao multiculturalismo ainda é incipiente. Há pesquisas que relacionam a criatividade ao bilinguismo (Mendonça \& Fleith, 2005; Zimmer \& Alves, 2014). Embora a proficiência em outra língua possa estar associada à experiência em culturas variadas, também pode existir sem que o indivíduo viva o multiculturalismo.

O estudo é ainda socialmente relevante em função da maior incidência do multiculturalismo, em suas diversas formas, no mundo atual. Com relação ao fluxo de migração internacional, por exemplo, o número de migrantes no mundo cresceu mais de $41 \%$ nos últimos 15 anos (Nações Unidas, 2015). Para além dos migrantes, há que se considerar seus familiares, que podem vivenciar o multiculturalismo dentro de casa, mesmo que nunca tenham vivido em outro país. Em um estudo envolvendo essa variável, realizado por Chang, Hsu, Shih e Chen (2014), foi examinada a criatividade em adolescentes filhos de indivíduos de diferentes nacionalidades. Os autores verificaram maiores índices de criatividade em jovens multiculturais quando comparados aos monoculturais.

Considerando que muito já foi descoberto sobre multiculturalismo e criatividade e que as oportunidades de trocas culturais têm se expan- dido, é mister realizar levantamento que apresente o estado da arte da referida problemática, no intuito de expor lacunas, sugerir futuras investigações e embasar estratégias de políticas públicas. Este estudo teve como objetivo mapear artigos publicados em periódicos, no período de 2010 a 2015, com o intuito de investigar a relação entre multiculturalismo e criatividade.

\section{Método}

Como estratégia de levantamento de material, três bases eletrônicas de dados foram consultadas, uma internacional e duas nacionais. Compuseram a pesquisa a base internacional PsycNET da American Psychological Association e as nacionais Scientific Electronic Library Online (SciELO) e Coordenação de Aperfeiçoamento de Pessoal de Nível Superior (CAPES).

Para a busca, foram utilizadas os pares de palavras-chave: criatividade e multiculturalismo e criatividade e biculturalismo, em português, e, em inglês, creativity e multiculturalism e creativity e biculturalism. Foram estabelecidos dois critérios de seleção: tipo de obra, centrando a busca apenas em artigos científicos publicados em periódicos, e data de publicação, delimitando o período entre 2010 e 2015.

A pesquisa nos bancos de dados foi realizada nos meses de outubro e novembro de 2015. Foram localizadas 126 publicações que continham as palavras pesquisadas, $38(30 \%)$ da base de dados da PsycNET, 85 (68\%) da base da CAPES e três (2\%) da SciELO. Em uma primeira conferência, foram aplicados os critérios de tipo e ano de publicação, eliminando-se 14 produções (11\%) que não se encaixavam na categorização de artigos publicados em periódicos e cinco (4\%) que foram publicados em anos anteriores a 2010. Outros 15 artigos (12\%) foram excluídos por estarem duplicados.

Assim, 92 produções científicas $(73 \%$ do total) tiveram seus resumos analisados com o objetivo de verificar a compatibilidade dos conteúdos com os objetivos desta revisão. A maioria deles foi retirada do banco da CAPES $(n=69)$, outros 14 artigos provinham do banco PsycNET 
e nove estavam disponíveis em ambas as bases de dados. Nenhum dos artigos analisados era da base SciELO.

\section{Procedimentos}

A partir da leitura dos resumos, buscou-se verificar se os 92 artigos identificados exploravam os dois construtos centrais deste estudo, multiculturalismo e criatividade, em seus objetivos. A maioria deles investigava apenas um dos construtos e, os demais, embora mencionassem os termos multiculturalismo e criatividade, tinham objetivos de pesquisa focados em outras temáticas. Foram excluídos 62 artigos (49\% do total).

Os 30 artigos remanescentes ( $24 \%$ do total) foram lidos na íntegra. Os dados provenientes dessa leitura foram catalogados em uma planilha com as seguintes categorias de análise: periódico, países de produção, tipo de autoria, ano de publicação, objetivos, tipo de estudo, abordagem metodológica do estudo, população pesquisada, instrumentos utilizados. Para análise dos periódicos, verificou-se o número de artigos publicados por revista. Buscou-se levantar também as áreas de conhecimento dos periódicos, por meio da leitura das descrições disponíveis em suas páginas da internet, e os idiomas nos quais os textos foram divulgados.

$\mathrm{Na}$ análise dos países de produção por artigo, foram observados os países em que se localizavam as universidades às quais os autores estavam vinculados, conforme descrito, em geral, nas notas do artigo. Por exemplo, no artigo assinado pelos autores Susannah B. F. Paletz, da Universidade de Maryland, Ella Miron-Spektor, do Instituto de Tecnologia de Israel, e Chun-Chi Lin, da Univerdade Nacional de Taiwan, foram registrados os países Estados Unidos (EUA), Israel e Taiwan. Nos casos de artigo em que mais de um autor ou mais de uma universidade eram de um mesmo país, este era contado apenas uma vez, já que o objetivo foi quantificar o número de publicações por país.

Para avaliar o tipo de autoria, foi verificado se os artigos eram de múltiplos autores ou de autoria individual. $\mathrm{O}$ ano de publicação foi catalogado conforme a data de publicação do periódico.
Para análise do tipo de estudo, foi verificado se as publicações tinham características de artigo empírico, revisão de literatura, artigo teórico, artigo metodológico ou estudo de caso. Utilizaram-se os critérios descritos pelo Manual da Associação Americana de Psicologia (American Psychological Association, 2010) para essa avaliação. De acordo com o Manual, estudos empíricos são relatórios de pesquisas com dados originais e geralmente contém as seções de introdução, método, resultados e discussão. Revisões de literatura são avaliações críticas de materiais que já foram publicados e têm características próximas às dos artigos teóricos, que são proposições de novas teorias ou análises de teorias existentes, ressaltando os avanços; ambos possuem estruturas parecidas, que não envolvem as seções comuns dos trabalhos empíricos. Artigos metodológicos são aqueles que apresentam novas abordagens metodológicas. Estudos de caso envolvem investigações de cenários com indivíduo, grupo, comunidade ou organização.

Nos artigos empíricos e nos estudos de caso, quando aplicável, foram analisadas ainda a abordagem e a amostra. $\mathrm{O}$ exame da abordagem considerou as categorias de estudo quantitativo, qualitativo ou ambos. Fleith e Costa (2005) ressaltaram que essa não é uma distinção simples. Para tanto, neste estudo, optou-se por seguir critérios baseados nas definições de Breakwell (1995). O autor descreveu como qualitativa a análise que descreve e detalha a natureza dos processos e quantitativa a análise que mede frequência e magnitude dos processos. A primeira está mais relacionada a significados e definições e a segunda à contagem e mensuração.

A população pesquisada foi avaliada com relação à faixa etária e classificada nas categorias: criança, adolescente, adulto estudante ou adulto profissional. Os participantes com idade de até 12 anos incompletos foram considerados crianças e com idade de 12 a 18 anos, adolescentes, conforme definição do Estatuto da Criança e do Adolescente (1990). Foram classificados adultos os participantes com idade acima de 18 anos, os estudantes de graduação ou de MBA e os demais profissionais. Como foi percebida uma distância 
entre a média etária dos estudantes e dos profissionais, os adultos foram classificados também quanto à ocupação.

Os instrumentos utilizados para mensurar criatividade e multiculturalismo foram analisa- dos apenas no caso de artigos empíricos. Nessa análise, catalogou-se a frequência de utilização e as autorias dos instrumentos. Alguns artigos continham relato de utilização de mais de um procedimento para medir criatividade ou multiculturalismo.

Tabela 1

Publicações sobre Criatividade e Multiculturalismo no Período de 2010 a 2015

\begin{tabular}{|c|c|c|}
\hline Publicação & Tipo de estudo & Países de produção \\
\hline Betz (2014) & Estudo de caso & Austrália \\
\hline Chang et al. (2014) & Empírico & Taiwan \\
\hline Chang, Su, \& Chen (2015) & Empírico & Taiwan \\
\hline Chao, Kung, \& Yao (2015) & Revisão de literatura & Canadá e China \\
\hline Cheng \& Leung (2012) & Empírico & Singapura \\
\hline Cheng et al. (2011) & Empírico & Singapura e Taiwan \\
\hline Cho \& Morris (2015) & Empírico & Estados Unidos \\
\hline Chua, Morris, \& Mor (2012) & Empírico & Estados Unidos \\
\hline Crisp \& Turner (2011) & Teórico & Inglaterra \\
\hline Crotty \& Brett (2012) & Empírico & Estados Unidos e Emirados Árabes Unidos \\
\hline Fee \& Gray (2012) & Empírico & Austrália \\
\hline Goclowska \& Crisp (2014) & Teórico & Holanda e Inglaterra \\
\hline Kharkhurin (2010) & Empírico & Emirados Árabes Unidos \\
\hline Klimczuk (2014) & Teórico & Polônia \\
\hline Krug (2013) & Estudo de caso & Nova Zelândia \\
\hline Lee \& Kim (2011) & Empírico & Estados Unidos \\
\hline Leung \& Chiu (2010) & Empírico & Singapura \\
\hline Leung \& Wang (2015) & Teórico & China \\
\hline Li et al. (2015) & Empírico & China e Taiwan \\
\hline Maddux, Adam, \& Galinsky (2010) & Empírico & Estados Unidos e França \\
\hline Moro (2014) & Empírico & França \\
\hline Nouri et al. (2013) & Empírico & Israel e Singapura \\
\hline Paletz, Miron-Spektor, \& Lin (2014) & Teórico & Estados Unidos, Israel e Taiwan \\
\hline Saad et al. (2012) & Empírico & Estados Unidos e Espanha \\
\hline Stahl, Makela, Zander, \& Maznevski (2010) & Revisão de literatura & Áustria, França, Finlândia, Suécia e Suíça \\
\hline Storme et al. (2015) & Empírico & China e França \\
\hline Tadmor, Galinsky et al. (2012) & Empírico & Estados Unidos e Israel \\
\hline Tadmor, Satterstrom, Jang, \& Polzer (2012) & Empírico & Estados Unidos e Israel \\
\hline Viki \& Williams (2014) & Empírico & Inglaterra \\
\hline Zhan, Bendapudi, \& Hong (2015) & Empírico & China e Singapura \\
\hline
\end{tabular}




\section{Resultados}

Os resultados foram analisados por meio de estatística descritiva, buscando mapear dados de publicação, autoria, objetivos e metodologia. Os 30 estudos, que tiveram seus textos analisados na íntegra, estão descritos na Tabela 1, com informações do tipo de estudo e o país de produção.

Foi observada uma prevalência dos artigos de autoria múltipla $(n=25)$ em comparação aos artigos de autoria individual $(n=5)$. A quantidade de publicações por ano tem se ampliado. Embora não haja um crescimento linear no número de artigos, pôde-se observar que essa temática foi mais investigada nos últimos três anos. Foram localizadas sete publicações por ano em 2014 e em 2015, número superior ao início do período pesquisado. Em 2010 foram quatro e, em 2011, três publicações. Entre 2012 e 2013, porém, houve uma oscilação significativa, com sete publicações no primeiro ano e apenas duas no segundo.

Observou-se preponderância de estudos empíricos ( $n=21$ artigos). Cinco estudos se encaixam na classificação de artigo teórico, dois de revisão de literatura e outros dois de estudos de caso. Nenhuma das publicações tinha características de estudo metodológico.

A nacionalidade de produção dos artigos foi bastante variada. Universidades de 18 diferentes países tiveram participação na produção dos artigos sobre criatividade e multiculturalismo. Os Estados Unidos foi o país que teve maior número de produções $(n=10)$. É forte a representatividade do continente asiático nessa análise, foram 21 publicações dessa região, sendo cinco da China, cinco de Singapura, cinco de Taiwan, quatro de Israel e dois dos Emirados Árabes Unidos. Quatorze artigos tinham autoria europeia e três eram da Oceania. Não foi identificada autoria do continente sul-americano, tampouco publicações em língua portuguesa. Quase todos os artigos foram escritos em língua inglesa $(n=29)$, e apenas um foi escrito em italiano, publicado na revista Interazioni.

Os 30 artigos analisados foram publicados em 21 periódicos. Grande parte das revistas, de diferentes áreas do conhecimento, só tiveram uma publicação. Foram identificadas publica- ções em revistas que se descreviam como das áreas de artes, ciências humanas, ciências sociais, filosofia, gestão, literatura e psicologia. Apenas quatro periódicos, da grande área psicologia, tiveram mais de uma publicação, são eles: Journal of Organizational Behavior $(n=4)$, Journal of Cross-Cultural Psychology $(n=4)$, The Journal of Creative Behavior $(n=3)$ e Social Psychological and Personality Science $(n=2)$.

Criatividade e multiculturalismo são construtos complexos, que podem ser pesquisados de diferentes maneiras. Nos artigos desta revisão, foi possível identificar a criatividade investigada nas perspectivas individual $(n=21)$, coletiva $(n=7)$ e em ambas ( $n=2)$. O construto multiculturalismo foi investigado na perspectiva individual ( $n=17)$; coletiva, como equipes, grupos e famílias $(n=8)$; e ainda como ambiente, sociedade, contexto ou comunidade multiculturais $(n=5)$.

Para conhecer melhor as pesquisas, fez-se uma análise dos objetivos de investigação. Buscou-se agrupar os trabalhos que tinham focos similares em categorias. Os propósitos identificados foram organizados em seis grupos: (a) explicar a relação entre multiculturalismo e criatividade por meio de mecanismos psicológicos; (b) debater ou comprovar a existência da relação ou correlação entre multiculturalismo e variáveis psicológicas, inclusive a criatividade; (c) analisar condições do ambiente que favorecem a criatividade; (d) discutir a relação do bilinguismo com a criatividade; (e) avaliar o impacto do tipo e estrutura da tarefa criativa em contextos multiculturais; (f) outros.

$\mathrm{Na}$ metade das publicações analisadas $(n=$ 15), os autores discutiram mecanismos subjacentes à relação do multiculturalismo com a criatividade. A identidade ou identificação cultural ( $n=$ 5), a metacognição cultural $(n=2)$, a percepção de distância cultural $(n=2)$, a aprendizagem sobre a nova cultura $(n=2)$, o estado emocional $(n=1)$, a percepção de conflito ou ameaça em ambientes multiculturais $(n=1)$, o processo de adaptação cognitiva $(n=1)$ e a capacidade de navegar em diferentes mind-sets $(n=1)$ foram estudados como meios ou estratégias para se desenvolver o potencial criativo diante da experiência multicultural. 
Uma investigação mais ampla foi realizada em seis artigos, nos quais buscou-se avaliar efeitos positivos e negativos do multiculturalismo em variáveis psicológicas e comportamentais, entre elas, a criatividade $(n=2)$ e evidenciar empiricamente a relação entre multiculturalismo e criatividade individual ou coletiva $(n=4)$. Em três publicações, os pesquisadores analisaram os elementos condicionantes do multiculturalismo para a promoção da criatividade. O clima para a inclusão $(n=1)$ e as condições públicas e institucionais em uma indústria $(n=1)$ foram estudados como mediadores da vivência em múltiplas culturas, além disso, foi realizada uma comparação entre a criatividade de indivíduos multiculturais em dois contextos socioculturais diferentes $(n=1)$.

Em dois artigos, o foco de estudo foi a relação do bilinguismo, fruto da experiência multicultural, com a criatividade. Autores de outros dois artigos debateram a importância da especificidade e da estrutura da tarefa criativa para o desempenho de equipes ou pessoas multiculturais. Três artigos tiveram objetivos díspares. Em um estudo avaliou-se a diferença da diversidade étnica e cultural em relação à inovação, em outro analisou-se a criatividade em uma obra literária produzida por um escritor com vivência multicultural. Um artigo trabalhou a perspectiva negativa da relação entre multiculturalismo e criatividade, tendo abordado o potencial do processo social negativo em criar obstáculos interculturais e consequentemente suprimir a criatividade de equipes com membros de culturas diversas.

Quanto à metodologia, avaliaram-se os tipos de abordagem e a amostra nos 21 artigos empíricos e nos dois estudos de caso. Analisando a abordagem, 19 produções tinham características de estudos quantitativos, dois apresentaram investigação qualitativa (ambos estudos de caso) e um era de abordagem mista, quantitativa e qualitativa. Com relação à amostra, foi possível observar que a maior parte dos artigos investigou adultos. Onze artigos contaram com participantes adultos universitários e quatro com adultos profissionais. Apenas três estudos pesquisaram crianças e dois pesquisaram adolescentes. Em três publicações, empíricas e de estudo de caso, não foi identificado o perfil dos participantes ou a pesquisa foi realizada por análise documental.
$\mathrm{Na}$ análise dos instrumentos foram considerados apenas os artigos empíricos de abordagem quantitativa $(n=19)$. Desse total, em $89 \%$ houve utilização de ferramenta para medir a criatividade, sendo que em $79 \%(n=15)$ foi aplicado pelo menos um instrumento previamente validado e em $37 \%(n=7)$ foi utilizado questionário ou tarefa original (elaborada pelo autor do estudo) para medir a criatividade. Em 10\% $(n=2)$ dos estudos foi utilizada apenas uma ferramenta para medir o construto, todos os demais empregaram mais de um procedimento quantitativo para analisar a criatividade.

Os testes de criatividade mais frequentes nas publicações foram os elaborados por Guilford e Torrance. O Teste de Usos Alternativos (Guilford, 1950, 1959, 1967) foi utilizado em cinco pesquisas. O Teste Torrance de Pensamento Criativo (Torrance, 1998), abreviado para adultos (Goff \& Torrance, 2002), ou adaptado para chineses, foi utilizado em cinco estudos.

O construto multiculturalismo, por outro lado, foi medido com instrumentos psicométricos em menos da metade dos artigos empíricos quantitativos. Nas demais publicações, os autores partiam da informação de que havia o multiculturalismo, por exemplo, no caso de participantes que viveram em diferentes países, sendo que isso era o bastante para caracterizá-los como multiculturais sem a utilização de outros métodos. Apenas $47 \%(n=9)$ dos artigos empíricos analisados buscaram medir esse construto. Dois instrumentos foram utilizados em mais de uma publicação, a Pesquisa de Experiência Multicultural ( $n=3$; Leung \& Chiu, 2010) e a Escala de Identidade Bicultural ( $n=2$; Benet-Martínez $\&$ Haritatos, 2005). Foram identificados instrumentos para medir a intensidade da exposição às multiculturas, a identificação dos sujeitos às culturas materna e hospedeira, o conhecimento sobre a nova cultura, o tipo de aculturação, a metacognição cultural e a distância entre as culturas.

\section{Discussão}

Um dos objetivos desta revisão foi conhecer e analisar como tem sido estudada a relação entre criatividade e multiculturalismo. A análise dos artigos sugere que, de uma forma geral, as 
variáveis estão interligadas. Destacam-se dois aspectos relevantes observados nos estudos desse campo: os mecanismos subjacentes ao desenvolvimento criativo diante do multiculturalismo e as condições que favorecem a relação entre os construtos. Além disso, foram identificados dois tópicos que podem indicar tendências para pesquisas futuras: as ferramentas de mensuração da criatividade e a definição do multiculturalismo.

Foi possível observar que a identificação cultural, por meio da adoção de medidas de aculturação (assimilação, separação, marginalização ou integração) e do biculturalismo, pode ser um processo essencial para que uma pessoa multicultural desenvolva a criatividade. Além disso, a percepção de distância entre culturas experienciadas, o aprendizado sobre a nova cultura, a metacognição cultural e a fluência em outro idioma também demonstraram mediar o desempenho criativo de sujeitos multiculturais.

As condições do contexto em que se dá o multiculturalismo podem influenciar os processos criativos. Pessoas com dupla filiação cultural responderam diferentemente aos testes de criatividade a depender do país em que estavam residindo (Kharkhurin, 2010). Em equipes de trabalho, observou-se que o clima para a inclusão é fundamental para permitir o compartilhamento de informações e consequentemente propiciar a criatividade (Li et al., 2015).

Sublima-se a importância da escolha do tipo e da estrutura de tarefas criativas empregada nas pesquisas e no cotidiano de instituições com pessoas multiculturais. Storme et al. (2015) verificaram que resultados encontrados usando uma tarefa específica de criatividade não podem ser generalizados para todos os tipos de criatividade. Ou seja, avaliar a criatividade verbal em uma tarefa de gerar muitas ideias (pensamento exploratório divergente) não é suficiente para concluir sobre criatividade geral de uma pessoa multicultural. Além disso, Nouri et al. (2013) verificaram que equipes culturalmente diversas beneficiam-se com instruções específicas quando desempenham uma tarefa executiva (convergente) e com instruções gerais quando estão desempenham uma tarefa criativa.

Assim, os resultados criativos de pessoas ou equipes multiculturais podem variar de acordo com o tipo e estrutura da tarefa realizada. Analisando os artigos empíricos localizados nesta revisão, foi possível observar que a maioria utilizou mais de um tipo de instrumento para medir a criatividade. Essa parece ser uma necessidade e uma tendência na investigação da criatividade em relação a efeitos da cultura.

Foi identificada uma grande variação nos conceitos que definiam os objetos de cada estudo, especialmente no que se refere ao entendimento do multiculturalismo. Essa variável foi descrita por alguns autores como múltiplas culturas dentro de um mesmo país. Para outros pesquisadores, o multiculturalismo aconteceria quando há interação entre diferentes culturas de duas ou mais nações. Em jovens, o multiculturalismo foi descrito em filhos que nascem e crescem em países diferentes dos pais. Houve ainda autores que estudaram o fenômeno a partir de duas identidades sociais inconsistentes, como mulher de negócios ou budista secular.

Além disso, foram investigadas diferentes dimensões do conceito. O multiculturalismo foi explorado como ambiente, indivíduo ou grupos, experiência e produto. Em algumas publicações não foi possível encontrar uma definição de multiculturalismo. Observa-se ainda que o emprego do termo multiculturalismo é amplo. Rudmin (2009), por exemplo, destaca que, no período entre 1918 e 2003, foram propostas mais de 100 taxonomias diferentes relativas a identidades culturais, entre elas o multiculturalismo. Para análise das transformações decorrentes da experiência multicultural de indivíduos ou grupos, Sam e Berry (2010) sugerem que o termo aculturação é mais apropriado do que multiculturalismo, assimilação, globalização e biculturalismo. É importante que estudos futuros delimitem a terminologia adotada ou que apresentem uma definição explícita do conceito de multiculturalismo aplicada.

Esta revisão objetivou também conhecer o estado da arte em pesquisas sobre criatividade e multiculturalismo em termos de quantidade, origem e tipologia de publicações. Os números demonstraram uma propensão crescente de artigos na área, especialmente com participantes adultos. Pôde-se perceber também uma continuidade nos objetivos de investigação ao longo do perí- 
odo investigado. Setenta e cinco por cento das pesquisas que buscaram evidenciar a relação entre multiculturalismo e criatividade, bilinguismo e outras variáveis psicológicas foram publicadas até 2012. De outro lado, os estudos mais recentes buscaram aprofundar o entendimento da relação entre os conceitos. Oitenta por cento dos artigos nos quais são discutidos mecanismos e condições subjacentes ao desenvolvimento criativo em multiculturais foi publicado a partir de 2012 .

No entanto, de forma geral, o número de artigos identificados foi relativamente baixo, considerando a importância do tema. As fronteiras nacionais estão mais fluidas e, cada vez mais, as pessoas se movimentam entre os países (Nações Unidas, 2015). Informações de pesquisa são essenciais para políticas e iniciativas de apoio (Flick, 2009), que podem contribuir para a inclusão e adaptação de pessoas multiculturais, favorecendo a criatividade (Li et al., 2015). É possível notar que há interesse dos pesquisadores por essas temáticas nas regiões de maior fluxo migratório.

De acordo com o Relatório de Migração Mundial (Nações Unidas, 2015), aproximadamente dois terços de todos os imigrantes do mundo vivem na Europa (76 milhões) ou na Ásia (75 milhões). Além de receber muitos migrantes, os continentes tiveram o maior número de pessoas que abandonaram suas origens para viver em outros países. Em 2015, Ásia e Europa representaram, respectivamente, a origem de $43 \%$ e $25 \%$ de todos os emigrantes do mundo (Nações Unidas, 2015). Isso poderia explicar o número de artigos identificados nesta revisão oriundos dos continentes asiático $(n=21)$ e europeu $(n=14)$.

Em uma comparação entre países, os Estados Unidos foi o que mais publicou sobre multiculturalismo e criatividade $(n=10)$. Não surpreende que os norte-americanos tenham especial interesse por matérias ligadas à miscigenação cultural. Os EUA, considerados a terra de oportunidades, ainda é o destino mais cobiçado no mundo. O país é, de longe, o que mais recebe migrantes internacionais no mundo. Em 2015, teve quase quatro vezes mais imigrantes que o segundo colocado da lista, a Alemanha (Nações Unidas, 2015).
O Brasil, quando comparado a outros países, não é considerado atualmente um forte polo de atração de imigração (Paiva \& Leite, 2014). Talvez por isso não tenha sido identificada nenhuma publicação do país com objetivo de investigar criatividade e multiculturalismo. No entanto, segundo Paiva e Leite, nos últimos anos a predominância de movimentação de pessoas na direção sul-norte vem se enfraquecendo, e países como o Brasil estariam despontando como polo receptor de estrangeiros. Fatores como o crescimento econômico, a inserção do país em organizações internacionais, os laços sociais com outros países e as políticas públicas brasileiras influenciariam o interesse pelo Brasil como destino de pessoas de outros países. Especialmente para refugiados, os atrativos do país têm, de fato, cativado.

De acordo com o relatório do Comitê Nacional para os Refugiados (2016), nos últimos cinco anos, o número de pedidos de refúgio cresceu $2.868 \%$ no Brasil, sendo que no final do ano de 2015 havia mais de 25 mil requerimentos de refugiados aguardando resposta, em sua maioria, de origem haitiana. Cabe refletir, portanto, sobre as expectativas de intercâmbio cultural nos próximos anos e sobre a importância de estudar o desenvolvimento criativo de pessoas que vivenciarão um processo aculturativo no Brasil.

A crise de refugiados, aliás, tem sido vivenciada em todo o mundo. De acordo com o relatório anual do Alto Comissariado das Nações Unidas para os Refugiados (2015), houve recorde de aumento anual no número refugiados no ano de 2014, em consequência dos conflitos territoriais, políticos e religiosos, tendo alcançado cerca de 60 milhões de pessoas. Nos últimos anos, o número de refugiados pelo mundo atingiu o nível mais alto desde a Segunda Guerra Mundial.

Trata-se de situação preocupante para governos e populações. À medida em que esses estrangeiros são integrados nas comunidades receptoras, soluções criativas podem transformar problemas em vantagens comparativas. De acordo com Alencar e Fleith (2009), a criatividade é um elemento de sobrevivência e de progresso. Nesse sentido, estudar a criatividade, fruto da experiência multicultural de refugiados, pode 
ser relevante. Apesar do aumento expressivo de refugiados e das condições críticas vivenciadas, nenhum dos 30 artigos identificados neste estudo investigou a criatividade relacionada ao multiculturalismo advindo da movimentação de refugiados. Esse, portanto, é um dos possíveis nichos promissores para futuras investigações.

Nos artigos analisados, sob o prisma da amostra estudada, foi observado um predomínio de participantes adultos, estudantes e profissionais. Isso pode estar relacionada ao fato de $72 \%$ dos migrantes internacionais ter idade entre 20 e 64 anos e apenas $15 \%$ ter menos de 20 anos (Nações Unidas, 2015). Esse número não deve, no entanto, ser visto isoladamente. A Organização das Nações Unidas ressalta em seu relatório que, embora a maioria das migrações ocorram em idade laboral, muitas crianças, filhos de migrantes, nascem nos países hospedeiros e não são consideradas nesse índice. Essas crianças estão inseridas em famílias multiculturais, como é o caso, por exemplo, do $44^{\circ}$ presidente dos EUA, Barack Obama, filho de queniano e norte-americana, que certamente vivenciou o multiculturalismo, embora seja cidadão norte-americano. Sugere-se dar mais atenção ao grupo de jovens migrantes de primeira ou segunda geração nos estudos dessa seara.

\section{Considerações Finais}

Tendo em vista os resultados encontrados, pode-se concluir que, de forma geral, criatividade e multiculturalismo são fenômenos interligados. Este é um tema de interesse de pesquisadores em diferentes lugares do mundo, especialmente nas regiões nas quais o fluxo migratório é maior. No Brasil, não foi identificada publicação. Há perspectivas de que nos próximos anos esse tema se torne mais representativo para o país, despertando o interesse dos pesquisadores.

Considerando o estado da arte no período de 2010 a 2015, sugere-se ampliar o estudo da criatividade com foco em crianças e adolescentes multiculturais. A escola, como ambiente de interação desse público, pode ser também investigada na perspectiva do desenvolvimento do potencial criativo. Além dos jovens, os refugiados, grupo que tem se crescido em função dos conflitos no mundo, foi um público negligenciado nas pesquisas e que pode ser um nicho relevante de investigação. Sugere-se ainda avaliar o emprego do termo multiculturalismo, considerando terminologias mais específicas de cada abordagem ou delimitando especificamente a definição do conceito. Como limitação do estudo, aponta-se a restrição a publicações em periódicos, tendo sido omitidas dissertações de mestrado e teses de doutorado.

\section{Referências}

Alencar, E. M. L. S., \& Fleith, D. S. (2009). Criatividade: Múltiplas perspectivas. Brasília, DF: Editora da Universidade de Brasília.

Alto Comissariado das Nações Unidas para os Refugiados. (2015). UNHCR Global trends 2014: Force displacement in 2014. Genebra: Author.

Amabile, T. M. (1996). Creativity in context. Update to the social psychology of creativity. Boulder, CO: Westview Press.

American Psychological Association. (2010). Manual de estilo da APA (6. ed.). Porto Alegre, RS: Artmed.

Aronowitz, M. (1984). The social and emotional adjustment of immigrant children. International Migration Review, 26, 89-110.

Benet-Martínez, V., Leu, J., Lee, F., \& Morris, M. W. (2002). Negotiating biculturalism: Cultural frame switching in biculturals with oppositional versus compatible cultural identities. Journal of Cross-Cultural Psychology, 33(5), 492-516. doi: 10.1177/0022022102033005005

Benet-Martínez, V., \& Haritatos, J. (2005). Bicultural identity integration (BII): Components and psychosocial antecedents. Journal of Personality, 73(4), 1015-1050. doi: 10.1111/j.14676494.2005.00337.x

Berry, J. W. (1990). Psychology of acculturation. In J. Berman (Ed.), Nebraska symposium on motivation: Cross-cultural perspectives. Current theory and research in motivation (pp. 201-234). Lincoln, NE: University of Nebraska Press.

Berry, J. W., Kim, U., \& Boski, P. (1987). Psychological acculturation of immigrants. In Y. K. Young \& W. B. Gudykunst (Eds.), Cross-cultural adaptation: Current theory and research (pp. 312-326). Newbury Park, CA: Sage. 
Berry, J. W., Kim, U., Minde, T., \& Mok, D. (1987). Comparative studies of acculturative stress. International Migration Review, 21(3), 491-511. doi: $10.2307 / 2546607$

Berry, J. W., \& Sam, D. L. (1997). Acculturation and adaptation. In J. W. Berry, M. H. Segall, \& C. Kagicibaci (Eds.), Handbook of cross-cultural psychology: Vol. 31. Social behaviour and application (pp. 291-326). Boston, MA: Allyn \& Bacon.

Betz, E. (2014). Polynesian youth hip hop: Intersubjectivity and Australia's multicultural audience. Ethnomusicology Forum, 23(2), 247-265. doi: http:// dx.doi.org/10.1080/17411912.2014.926630

Breakwell, G. M. (1995). Research: Theory and methods. In G. M. Breakwell, S. Hammond, \& C. Fife-Schow (Eds.), Research methods in psycho$\log y$ (pp. 5-15). Londres: Sage.

Chang, J. H., Hsu, C. C., Shih, N. H., \& Chen, H. C. (2014). Multicultural families and creative children. Journal of Cross-Cultural Psychology, 45(8), 1288-1296. doi: $10.1177 / 0022022114537556$

Chang, J. H., Su, J. C., \& Chen, H. C. (2015). Cultural distance between parents' and children's creativity. Cultural Diversity and Ethnic Minority Psychology, 21(3), 477-485. doi: 10.1037/ a0037539

Chao, M. M., Kung, F. Y. H., \& Yao, D. J. (2015). Understanding the divergent effects of multicultural exposure. International Journal of Intercultural Relations, 47(2), 78-88. doi: 10.1016/j. ijintrel.2015.03.032

Cheng, C. Y., \& Leung, A. K. (2012). Revisiting the multicultural experience-creativity link: The effects of perceived cultural distance and comparison mind-set. Social Psychological \& Personality Science, 4(4), 475-482. doi: $10.1177 / 1948550612462413$

Cheng, C.-Y., Leung, A. K.-Y., \& Wu, T.-Y. (2011). Going beyond the multicultural experiencecreativity link: The mediating role of emotions. Journal of Social Issues, 67(4), 806-824. doi: 10.1111/j.1540-4560.2011.01729.x

Cheng, C.-Y., Sanchez-Burks, J., \& Lee, F. (2008). Connecting the dots within: Creative performance and identity integration. Psychological Science, 19(1), 1177-1183. doi: 10.1111/j.14679280.2008.02220.x.
Cho, J., \& Morris, M. W. (2015). Cultural study and problem-solving gains: Effects of study abroad, openness, and choice. Journal of Organizational Behavior, 36(7), 944-966. doi: 10.1002/job.2028

Chua, R. Y. J., Morris, M. W., \& Mor, S. (2012). Collaborating across cultures: Cultural metacognition and affect-based trust in creative collaboration. Organizational Behavior and Human Decision Processes, 118(2), 116-131. doi: http:// dx.doi.org/10.1016/j.obhdp.2012.03.009

Comitê Nacional para os Refugiados. (2016). Sistema de Refúgio Brasileiro: Desafios e perspectivas. Brasília, DF: Ministério da Justiça. Recuperado em http://www.acnur.org/fileadmin/scripts/doc. php?file=fileadmin/Documentos/portugues/Estatisticas/Sistema_de_Refugio_brasileiro_-_Refugio_em_numeros_-_05_05_2016

Crisp, R. J., \& Turner, R. N. (2011). Cognitive adaptation to the experience of social and cultural diversity. Psychological Bulletin, 137(2), 242266. doi: $10.1037 / \mathrm{a} 0021840$

Crotty, S. K., \& Brett, J. M. (2012). Fusing creativity: Cultural metacognition and teamwork in multicultural teams. Negotiation and Conflict Management Research, 5(2), 210-234. doi: 10.1111/j.1750-4716.2012.00097.x

Estatuto da Criança e do Adolescente. (1990). Brasília, DF: Presidência da República.

Fee, A., \& Gray, S. J. (2012). The expatriate-creativity hypothesis: A longitudinal field test. Human Relations, 65(12), 1515-1538. doi: 10.1177/0018726712454900

Fleith, D. S., \& Costa, A. L., Jr. (2005). Métodos de pesquisa em psicologia do desenvolvimento: $\mathrm{O}$ que é relevante considerar. In M. A. Dessen \& A. L. Costa Junior (Eds.), A ciência do desenvolvimento humano: Tendências atuais e perspectivas futuras (pp. 37-49). Porto Alegre, RS: Artmed.

Flick, U. (2009). Introdução à pesquisa qualitativa (3. ed.). São Paulo, SP: Artmed.

Friedman, R., \& Liu, W. (2009). Biculturalism in management: Leveraging the benefits of intrapersonal diversity. In R. S. Wyer, C.-Y. Chiu, \& Y.-Y. Hong (Eds.), Understanding culture: Theory, research, and application (pp. 343-360). New York: Psychology Press.

Guilford, J. P. (1950). Creativity. American Psychologist, 5(9), 444-454. doi: 10.1037/h0063487 
Guilford, J. P. (1959). Traits of creativity. In H. H. Anderson (Ed.), Creativity and its cultivation (pp. 142-161). New York: Harper.

Guilford, J. P. (1967). The nature of human intelligence. New York: McGraw-Hill.

Goclowska, M. A., \& Crisp, R. J. (2014). How dual-identity processes foster creativity. Review of General Psychology, 18(3), 216-236. doi: $10.1037 /$ gpr0000008

Goff, K., \& Torrance, E. P. (2002). Abbreviated Torrance Test for Adults. Bensenville, IL: Scholastic Testing Service.

Inglis, C. (1996). Multiculturalism: New policy responses to diversity. Paris: United Nations Educational, Scientific and Cultural Organization.

Kharkhurin, A. V. (2010). Sociocultural differences in the relationship between bilingualism and creative potential. Journal of CrossCultural Psychology, 41(5), 776-783. doi: $10.1177 / 0022022110361777$

Klimczuk, A. (2014). Barriers to the development of creative industries in culturally diverse region. Coactivity: Philosophy, Communication, 22(2), 145-152. doi: $10.3846 /$ cpc.2014.13

Krug, M. (2013). International creation examining cross-cultural influences in Ernest Hemingways for whom the bell tolls. Interdisciplinary Literary Studies, 15(2), 261-288.

Kuo, B. C. H. (2014). Coping, acculturation, and psychological adaptation among migrants: A theoretical and empirical review and synthesis of the literature. Health Psychology and Behavioral Medicine, 2, 16-33. doi: 10.1080/21642850.2013.843459

Lee, H., \& Kim, K. H. (2011). Can speaking more languages enhance your creativity? Relationship between bilingualism and creative potential among Korean American students with multicultural link. Personality and Individual Differences, 50(8), 1186-1190. doi:10.1016/j. paid.2011.01.039

Leung, A. K.-Y., \& Chiu, C.-Y. (2010). Multicultural experience, idea receptiveness, and creativity. Journal of Cross-Cultural Psychology, 41(5), 723-741. doi: 10.1177/0022022110361707

Leung, K., \& Wang, J. (2015). Social processes and team creativity in multicultural teams: A sociotechnical framework. Journal of Organizational Behavior, 36(7), 1008-1025. doi: 10.1002/ job.2021
Li, C.-R., Lin, C.-J., Tien, Y.-H., \& Chen, C.-M. (2015). A multilevel model of team cultural diversity and creativity: The role of climate for inclusion. The Journal of Creative Behavior, 1-22. doi: 10.1002/jocb.93

Maddux, W. W., Adam, H., \& Galinsky, A. D. (2010). When in Rome ... Learn why the Romans do what they do: How multicultural learning experiences facilitate creativity. Personality and Social Psychology Bulletin, 36(6), 731-741. doi: $10.1177 / 0146167210367786$

Malzberg, B., \& Lee, E. (1956). Immigration and mental disease. New York: Social Science Research Council.

Mendonça, P. V. C. F., \& Fleith, D. S. (2005). Relação entre criatividade, inteligência e auto-conceito em alunos monolingues e bilíngües. Psicologia Escolar e Educacional, 9(1), 59-70.

Mok, A., \& Morris, M. W. (2012). Managing two cultural identities: The malleability of bicultural identity integration as a function of induced global or local processing. Personality and Social Psychology Bulletin, 38(2), 233-246. doi: $10.1177 / 0146167211426438$

Mok, A., Morris, M. W., Benet-Martínez, V., \& Karaki Tapoglu-Aygun, Z. (2007). Embracing American culture: Structures of social identity and social networks among first-generation biculturals. Journal of Cross-Cultural Psychology, 38(5), 629-635.

Moro, M. R. (2014). Risk and creativity in adolescence within a multicultural society. Interazioni, 1, 21-31.

Nações Unidas. (2015). International Migration Report 2015: Highlights. New York: Author.

Nakano, T. C. (2005). Pesquisa em criatividade: Análise da produção científica do banco de teses da Capes (1996-2001). In G. P. Witter (Ed.), Metaciência e Psicologia (pp. 35-48). Campinas, SP: Alínea.

Nakano, T. C., \& Wechsler, S. M. (2007). Criatividade: Características da produção científica brasileira. Avaliação Psicológica, 6(2), 261-270.

Nouri, R., Erez, M., Rockstuhl, T., Ang, S., LeshemCalif, L., \& Rafaeli, A. (2013). Taking the bite out of culture: The impact of task structure and task type on overcoming impediments to crosscultural team performance. Journal of Organizational Behavior, 34(6), 739-763. doi: 10.1002/ job. 1871 
Paiva, A. L. B., \& Leite, A. P. M. R. (2014). Da emigração à imigração? Por uma análise do perfil migratório brasileiro nos últimos anos. Ars Histórica, 7, 1- 20.

Paletz, S. B. F., Miron-Spektor E., \& Lin, C. (2014). A cultural lens on interpersonal conflict and creativity in multicultural environments. Psychology of Aesthetics, Creativity, and the Arts, 8(2), 237-252. doi: 10.1037/a0035927

Rudmin, F. W. (2009). Catalogue of acculturation constructs: Descriptions of 126 taxonomies, 1918-2003. Online Readings in Psychology and Culture, 8(1). doi: 10.9707/ 2307-0919.1074

Saad, C. S., Damian, R. I., Benet-Martínez, V., Moons, W. G., \& Robins, R. W. (2012). Multiculturalism and creativity: Effects of cultural context, bicultural identity, and ideational fluency. Social Psychological and Personality Science, 4(3), 369-375. doi: 10.1177/1948550612456560

Sam, D. L., \& Berry, J. W. (2010). Acculturation: When individuals and groups of different cultural backgrounds meet. Perspectives on Psychological Science, 5, 472-481. doi: $10.1177 / 1745691610373075$

Stahl, G. K., Makela, K., Zander, L., \& Maznevski, M. L. (2010). A look at the bright side of multicultural team diversity. Scandinavian Journal of Management, 26(4), 439-447. doi: 10.1016/j. scaman.2010.09.009

Storme, M., Lubart, T., Myszkowski, N., Cheung, P. C., Tonge, T., \& Lau, S. (2015). A cross-cultural study of task specificity in creativity. The Journal of Creative Behavior, 1-15. doi: 10.1002/ jocb. 123

Tadmor, C. T., Galinsky, A. D., \& Maddux, W. W. (2012). Getting the most out of living abroad: Biculturalism and integrative complexity as key drivers of creative and professional success. Journal of Personality and Social Psychology, 103(3), 520-542. doi: 10.1037/a0029360

Tadmor, C. T., Hong, Y.-Y., Chao, M. M., Wiruchnipawan, F., \& Wang, W. (2012). Multicultural experiences reduce intergroup bias through epistemic unfreezing. Journal of Personality and Social Psychology, 103(5), 750-772. doi: 10.1037/a0029719
Tadmor, C. T., Satterstrom, P., Jang, S., \& Polzer, J. T. (2012). Beyond individual creativity: The superadditive benefits of multicultural experience for collective creativity in culturally diverse teams. Journal of Cross-Cultural Psychology, 43(3), 384-392.

Torrance, E. P. (1998). The Torrance tests of creative thinking norm-technical manual figural (streamlined) forms $A \& B$. Bensenville, IL: Scholastic Testing Service.

Viki, G. T., \& Williams, M. L. J. (2014). The role of identity integration in enhancing creativity among mixed-race individuals. The Journal of Creative Behavior, 48(3), 198-208. doi: 10.1002/jocb.48

Wechsler, S. M., \& Nakano, T. C. (2003). Produção brasileira em criatividade: $\mathrm{O}$ estado da arte. Escritos sobre Educação, 2(2), 43-50.

Witter, G. P. (1999). Metaciência e leitura. In G. P. Witter (Ed.), Leitura: Textos e pesquisas (pp. 13-22). Campinas, SP: Alínea.

Yoon, E., Chang, C. T., Kim, S., Clawson, A., Cleary, S. E., Hansen, M., ...Gomes, A. (2012). A metaanalysis of acculturation/enculturation and mental health. Journal of Counseling Psychology, 60, 1-16. doi: 10.1037/a0030652

Zanella, A. V., \& Titon, A. P. (2005). Análise da produção científica sobre criatividade em programas brasileiros de pós-graduação em psicologia. Psicologia em Estudo, 10(2), 305-316. doi: $10.1590 / \mathrm{S} 1413-73722005000200018$

Zhan, S., Bendapudi, N., \& Hong, Y-Y. (2015). Re-examining diversity as a double-edged sword for innovation process. Journal of Organizational Behavior, 36(7), 1026-1049. doi: 10.1002/ job.2027

Zimmer, M. C., \& Alves, U. K. (2014). O impacto do bi/multilinguismo sobre o potencial criativo em sala de aula: Uma abordagem via teoria dos sistemas dinâmicos. Educação e Contemporaneidade, 23(41), 77-89.

Recebido: $1 \% 1 / 2017$

$1^{a}$ revisão: $24 / 03 / 2017$ Aceite final: $31 / 05 / 2017$ 